\title{
Morphology in middle school
}

\section{HOLGER JUUL, ANNE-METTE VEBER NIELSEN AND HANNE TREBBIEN DAUGAARD}

Word learning in school children is thought to occur, to a large degree, during independent reading (Cunningham 2005). One way of learning a newly encountered word is to recognize its morphological components and derive a possible meaning from these. Hence, it is likely that word learning can be stimulated through instruction in morphological analysis (Baumann et al. 2003). With this in view, we designed an experimental teaching program for middle grade Danish students (grade 4) as part of an on-going project. Key questions have been what the morphology program should focus on in particular if Danish students are to benefit from it: Do middle grade students encounter morphologically complex words at all during independent reading? What types of complexities are they most likely to encounter? Are the words semantically transparent? We sought answers by examining 359 so-called Tier 2 words (Beck and McKeown 1985) from texts aimed at the middle grades. Tier 2 words are an appropriate target because they constitute a layer in-between the most basic vocabulary and domain specific words. We found that $85 \%$ of the words were morphologically complex, and that derivations (especially derivations by suffix) were the dominating type. We estimated that about one third were semantically transparent and that another third were partly transparent. Building on these results, the main elements of the teaching program are outlined. 